\title{
Quality assessment of resistance spot welding joints of AISI 304 stainless steel based on elastic nets
}

\author{
Óscar Martín ${ }^{a, *}$, Virginia Ahedo ${ }^{b}$, José Ignacio Santosc ${ }^{c}$ Pilar De Tiedra ${ }^{d}$, José Manuel Galán ${ }^{c}$ \\ a Ingeniería de los Procesos de Fabricación, Departamento CMeIM/EGI/ICGF/IM/IPF, Universidad de \\ Valladolid, Escuela de Ingenierías Industriales, Paseo del Cauce 59, Valladolid 47011, Spain. \\ b CaSEs - Complexity and socioecological systems, Depto. Arqueología y Antropología. Institución Milá y \\ Fontanals - CSIC. C/ Egipcíaques, 15. 08001 Barcelona, Spain. \\ c INSISOC, Área de Organización de Empresas, Departamento de Ingeniería Civil, Escuela Politécnica \\ Superior, Universidad de Burgos, Edificio La Milanera, C/Villadiego S/N, Burgos 09001, Spain. \\ d Ciencia de los Materiales e Ingeniería Metalúrgica, Departamento CMeIM/EGI/ICGF/IM/IPF, \\ Universidad de Valladolid, Escuela de Ingenierías Industriales, Paseo del Cauce 59, Valladolid 47011, \\ Spain. \\ * Corresponding author. Ph.: +34-983423533, Fax: +34-983423310, E-mail: oml@eii.uva.es (Ó. Martín)
}

\begin{abstract}
In this work, the quality of resistance spot welding (RSW) joints of 304 austenitic stainless steel (SS) is assessed from its tensile shear load bearing capacity (TSLBC). A predictive model using a polynomial expansion of the relevant welding parameters, i.e. welding current (WC), welding time (WT) and electrode force (EF) and elastic net regularization is proposed. The predictive power of the elastic net approach has been compared to artificial neural networks (ANNs), previously used to predict TSLBC, and smoothing splines in the framework of a generalized additive model. The results show that the predictive and classification error of the elastic net model are statistically comparable to benchmarks of the best pattern recognition tools whereas it overcomes correlation problems and performs variable selection at the same time, resulting in a simpler and more interpretable model. These features make the elastic net model amenable to be used in the design of welding conditions and in the control of the manufacturing processes.
\end{abstract}

Keywords: Resistance spot Welding; AISI 304 stainless steel; tensile shear load bearing capacity; quality assessment; elastic nets; smoothing splines.

\section{Introduction}

Resistance Spot Welding (RSW) is, according to Becker et al. [1], one of the primary methods to join sheet metals for automotive components due to the fact that, as indicated by Khodabakhshi et al. [2], it has the highest throughput.. In addition to the automotive industry, RSW of stainless steel (SS) sheets is also widely used, as pointed out by Kianersi at al. [3], in transportation vessels, home and office items, kitchen furniture and utensils and building applications. Feng et al. [4] stated that 4000-6000 RSW joints are used in each vehicle; as 
emphasised by Martín et al. [5], such a large number of RSW joints makes attractive the use of tools capable of reliably assessing the quality of RSW joints from its welding parameters that, thus, allow, as mentioned by Pereda at al. [6]: (i) warning in real time about potentially detrimental drifts in the RSW process; and (ii) assisting directly in quality control of the RSW process, reducing post-welding testing.

Özyürek [7] indicated that structures employing RSW joints are usually designed so that these joints are loaded in shear when the parts are exposed to tension or compression loading. Zhou et al. [8] reported that static tensile shear test is the most common laboratory test used in the determination of weld strength because of its simplicity. Thus, in the present work, the quality of the RSW joints is assessed from its tensile shear load bearing capacity (TSLBC), which is the peak load value obtained during the tensile shear test. Hasanbaşoğlu and Kaçar [9] and Kong et al. [10] agreed that the most important factor affecting TSLBC is the size of weld nugget, which, as explained by Raoelison et al. [11], is formed from the solidification of the molten metal after a heating by Joule effect.

Some previous works have already developed tools for assessing the effect of RSW parameters on welding quality. Identifying the most appropriate approach to build a predictive model is a challenging task. Wolpert [12] showed that there is no learning algorithm better than all the others on all the contexts. Consequently, it is necessary to run computational experiments in order to find out which are the techniques with the best performance for the particular case under consideration. Martín et al. [13] created a tool based on ANNs for the classification of ultrasonic oscillograms obtained from RSW joints. Li [14] carried out a fault diagnosis method in manufacturing processes using a functional regression approach. Moshayedi and Sattari-Far [15] proposed a finite element model to investigate the distribution of temperature and nugget formation during RSW process, as well as to study the effect of welding current (WC) and welding time (WT) on weld nugget size. Ma and Murakawa [16] studied the weld nugget formation process by using a finite element model which considered the coupling of the electrical field, thermal field and mechanical field during RSW process. Han et al. [17] used statistical models to study several forms of estimating the mechanical strength of RSW joints. Luo et al. [18] monitored in real-time the change of WC and electrode voltage in the secondary circuit and, thus, the dynamic resistance across electrodes was used to characterize the weld nugget growth. Martín et al. [19] developed a model based on artificial neural networks (ANNs) to predict the TSLBC of RSW joints from WT, WC and electrode force (EF) but with the drawback that ANNs are "black boxes", i.e. they lack explanatory power. Therefore, as pointed out by Martín et al. (2014), the underlying knowledge captured by the network during its training is not transparent to the user and, consequently, ANNs do not offer any interpretability of the results.

Depending on the purpose of the model, this issue can be relevant for model selection. Predictive accuracy is a common criterion for selecting a model. However, as pointed out by several authors [21,22], model simplicity and interpretability make it significantly easier to move from pattern recognition to knowledge extraction, that may be more useful to define, control and optimize industrial processes. In these cases, decision tools are more likely to be accepted if the results can be understood and explained [23], which means that among 
different models with predictive accuracy rates not statistically different in terms of a given significance, the simpler and more interpretable model will be preferred.

Unlike ANNs, regression techniques do offer interpretability of the results. Cho and Rhee [24] proposed simple linear and non-linear regression models to estimate weld strength and nugget diameter of RSW joints of low-carbon steel sheets, comparing the obtained results with those of ANNs. They found better prediction accuracy for ANNs.

A common approach to improve the performance of linear regression approaches capturing non-linear effects consists on obtaining extra regressors from the initial predictors, for instance, by using polynomial expansions. However, this procedure is not without its drawbacks. Predictors obtained this way are very correlated, the complexity and interpretability of the model increases, and there is an important risk of overfitting.

The elastic net regularization method proposed by Zou and Hastie [25] is used in this work to simultaneously obtain an interpretable and accurate predictive model. This approach produces simple and interpretable models while maintaining a good performance (even in the presence of several highly correlated variables), by means of reducing the number of predictors, identifying the most important ones and shrinking coefficients. In the present study, different polynomial expansions are implemented and compared to the performance of ANNs, previously used for this problem and with smoothing splines, a very flexible, although not interpretable, regression approach. The differences among the results were found not statistically significant. The simple, accurate and interpretable regression model obtained by applying elastic net regularization makes easier the design and optimization of the welding operation conditions and the control of the manufacturing process while its predictive accuracy is statistically comparable to that of the black box techniques. Additionally, the obtained model was analysed as a binary quality classification tool. Again, the performance of the model used as a classifier is competitive compared to the best welding pattern recognition algorithms found for welding quality control.

The structure of the paper is as follows: First of all, the experimental procedure is described. Initially, the composition and material properties are analysed in detail. Then, a description of the welding conditions as well as an explanation of the test selected to assess the quality of each spot is given. The next section presents the different data analysis methods studied, focusing at first on theoretical aspects of the different techniques and on the framework of comparison of all of them. Afterwards, the results and discussion are provided for both prediction and classification. The last section is devoted to the conclusions.

\section{Experimental Procedure}

\subsection{Materials and equipment}

The chemical composition and the mechanical properties of the AISI 304 austenitic SS sheets welded by RSW are, respectively, shown in Tables 1 and 2 . The sheet thickness was $0.8 \mathrm{~mm}$. 
Table 1. Chemical composition of the AISI 304 austenitic SS sheets (wt. \%).

\begin{tabular}{cccccccc}
\hline $\mathrm{C}$ & $\mathrm{Cr}$ & $\mathrm{Ni}$ & $\mathrm{Si}$ & $\mathrm{Mn}$ & $\mathrm{Mo}$ & $\mathrm{Al}$ & $\mathrm{Co}$ \\
\hline 0.08 & 18.03 & 8.74 & 0.426 & 1.153 & 0.36 & 0.003 & 0.17 \\
\hline $\mathrm{Cu}$ & $\mathrm{Nb}$ & $\mathrm{Ti}$ & $\mathrm{V}$ & $\mathrm{W}$ & $\mathrm{S}$ & $\mathrm{P}$ & $\mathrm{Fe}$ \\
\hline 0.39 & 0.02 & 0.004 & 0.05 & 0.03 & 0.002 & 0.019 & Bal. \\
\hline
\end{tabular}

Table 2. Mechanical properties of the AISI 304 austenitic SS sheets.

\begin{tabular}{cccc}
\hline $\begin{array}{c}\text { Yield strength } \\
(\mathrm{MPa})\end{array}$ & $\begin{array}{c}\text { Tensile strength } \\
(\mathrm{MPa})\end{array}$ & $\begin{array}{c}\text { Total elongation } \\
(\%)\end{array}$ & $\begin{array}{c}\text { Microhardness } \\
(\mathrm{HV}, 100 \mathrm{~g})\end{array}$ \\
\hline 290 & 675 & 70 & 162 \\
\hline
\end{tabular}

The AISI 304 austenitic SS sheets were welded with a single-phase alternating current (AC) 50 $\mathrm{Hz}$ equipment by using water-cooled truncated cone RWMA Group A Class 2 electrodes with $16 \mathrm{~mm}$ body diameter and $4.5 \mathrm{~mm}$ face diameter.

\subsection{Welding of the tensile shear test specimens}

The controlled parameters in the RSW process were: (i) WT that varied from 12 to 2 cycles, with a 1 cycle step decrease; (ii) WC that varied approximately from 6.5 to 1.5 kA RMS with a 0.5 kA RMS step decrease; and (iii) EF that took only two values: 1000 and $1500 \mathrm{~N}$. These three parameters are, as stated by Aslanlar [26], the most important welding parameters in RSW.

Thus, there were 242 , i.e. $11 \times 11 \times 2$, different welding conditions and a tensile shear test specimen was spot welded for each of these 242 welding conditions. The tensile shear test specimens were prepared according to [27] (see Martín et al. [19] for more details).

The weld nugget of the RSW joint is a cast dendritic microstructure with coarser grains than the polygonal austenitic grains of the adjacent metal, as shown in Fig.1. 


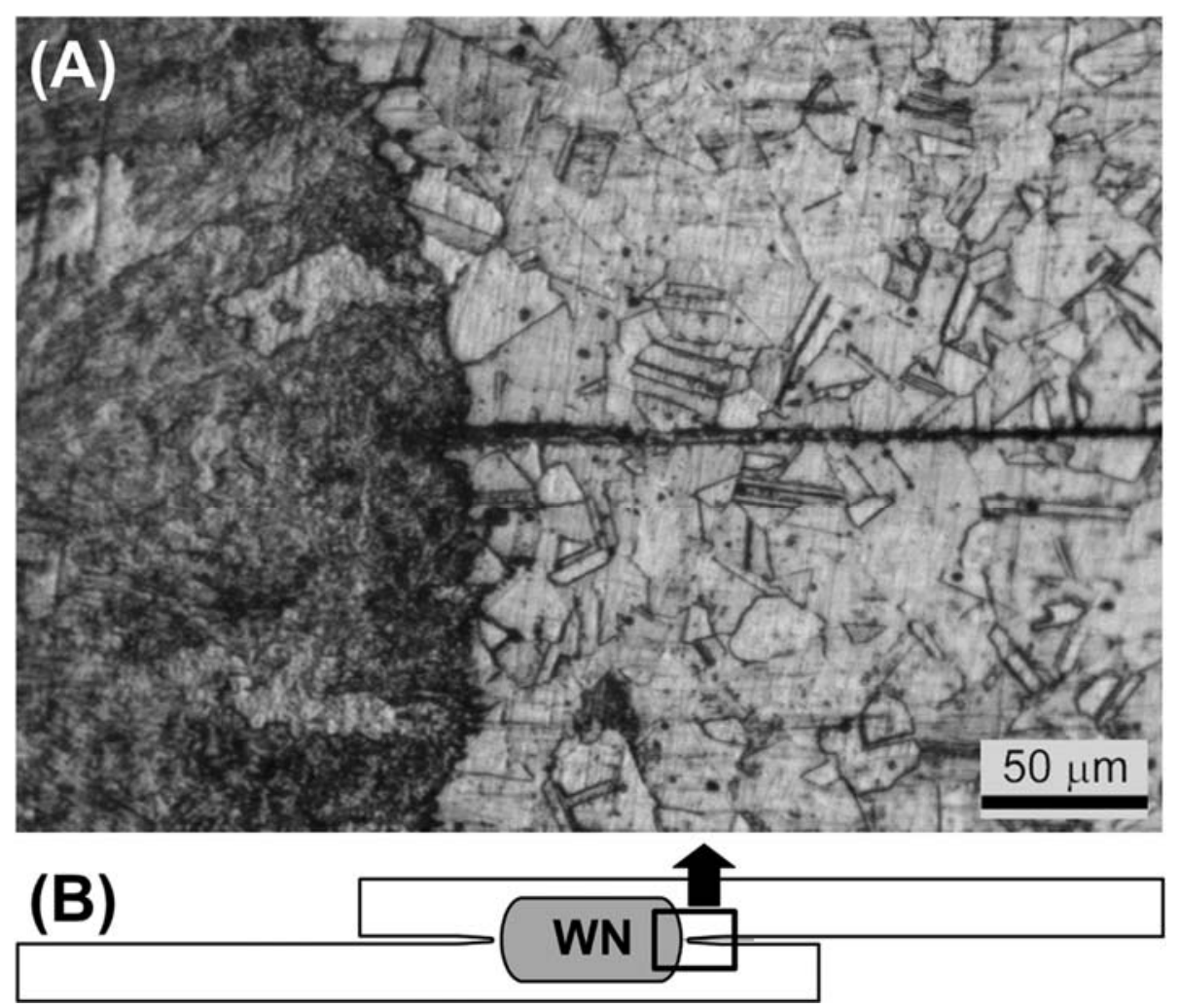

Fig. 1. (A) Micrograph that shows the cast dendritic microstructure of the weld nugget (WN) with coarser grains than the polygonal austenitic grains of the adjacent metal; electrolytic etching with oxalic acid according to Practice $A$ of [28]. (B) Diagram (not to scale) that shows the location of the area where the micrograph (A) was taken from the tensile shear test specimen.

\subsection{Quality assessment from TSLBC values}

A TSLBC value was obtained from each of the 242 tensile shear tests that were performed at a crosshead speed of $2 \mathrm{~mm} / \mathrm{min}$, which, according to Marashi et al. [29], allows to consider the test as static.

The minimum acceptable TSLBC value was set at $5.93 \mathrm{kN}$ and, therefore, the RSW joints whose TSLBC value was: (i) equal to or greater than $5.93 \mathrm{kN}$, were considered acceptable; (ii) less than $5.93 \mathrm{kN}$, were considered unacceptable. This criterion was based on the weld nugget diameter recommended by JIS Z 3140 [30]:

$$
d \geq 5 \sqrt{t}
$$

where $d$ is the weld nugget diameter and $t$ is the sheet thickness; thus, the minimum TSLBC obtained in the tensile shear tests for an acceptable RSW joint, which according to Eq. (1) has a weld nugget diameter equal to or greater than $4.5 \mathrm{~mm}$, was $5.93 \mathrm{kN}$.

This standard [30] also established an acceptance criterion based on the TSLBC itself, where, for a sheet thickness of $0.8 \mathrm{~mm}$ and since the tensile strength of the base metal exceeded 590 $\mathrm{MPa}$, the recommended value was a TSLBC equal to or greater than $5.65 \mathrm{kN}$. Thus, the 
criterion based on the weld nugget diameter was chosen because it was more conservative than that based on the TSLBC itself.

The weld nugget diameter of the RWS joints was evaluated by using ultrasonic testing. Since, as reported by Mansour [31], the ultrasonic beam width must be approximately equal to the smallest allowable weld nugget diameter which, according to Eq. (1), was set at $4.5 \mathrm{~mm}$, the selected transducer diameter was $4.5 \mathrm{~mm}$. The transducer, whose frequency was $20 \mathrm{MHz}$, used a captive water column delay and a replaceable rubber membrane with the aim of achieving good coupling with the surface of the RSW joint.

An acceptable RSW joint has a weld nugget whose diameter is greater than the ultrasonic beam width; hence, as Fig. 2 shows: (i) the span of the sequence of echoes is short due to the high attenuation caused by the coarse cast dendritic microstructure of the weld nugget; and (ii) the distance between consecutive echoes is the sum of the thickness of the two sheets because the ultrasonic beam reflections occur at the bottom surface of the lower sheet.

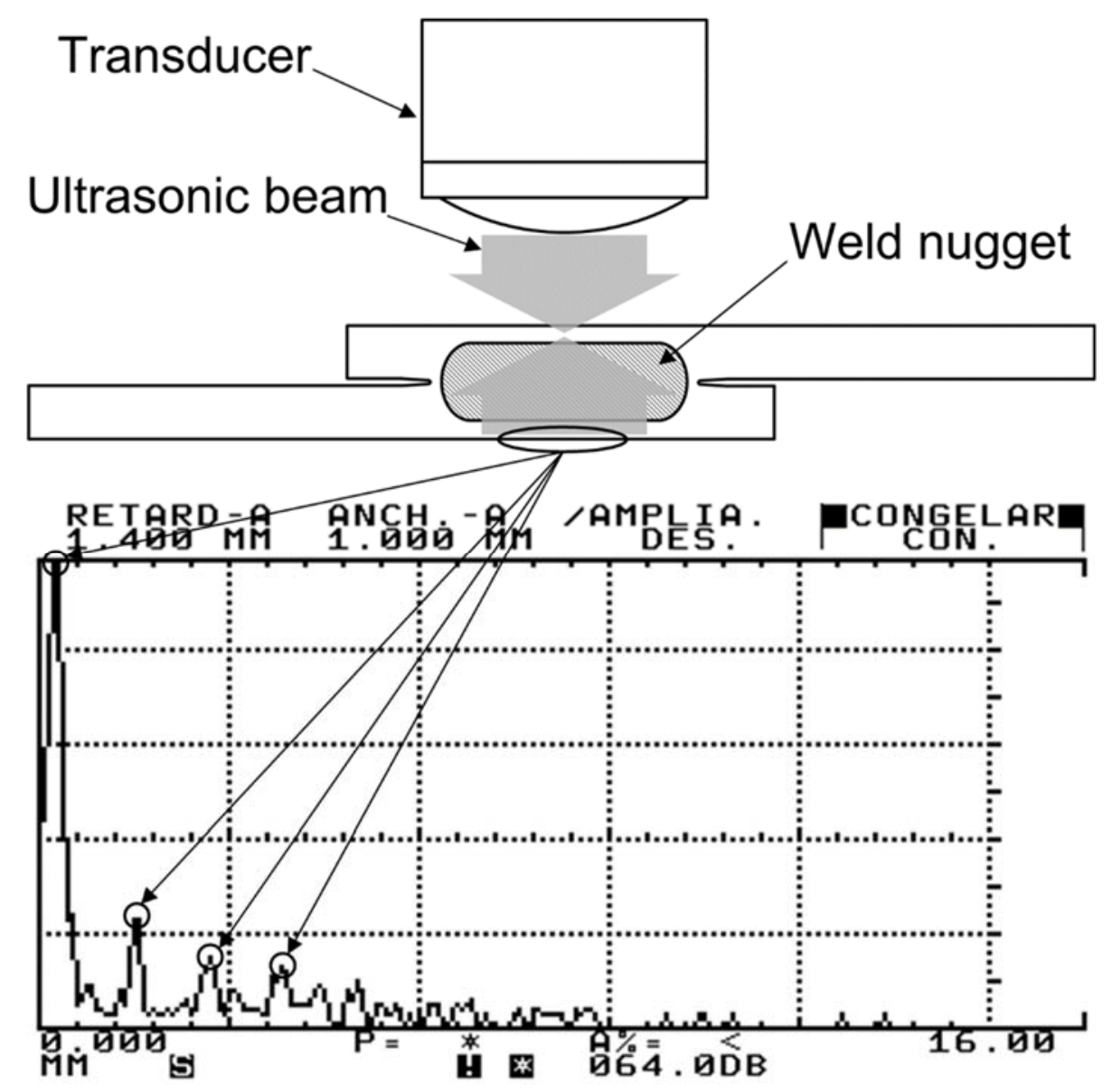

Fig. 2. Top: reflections of the ultrasonic beam for an acceptable RSW joint in a tensile shear test specimen (not to scale); bottom: ultrasonic oscillogram of an acceptable RSW joint.

An unacceptable RSW joint has a weld nugget whose diameter is smaller than the ultrasonic beam width; therefore, as Fig. 3 shows: (i) the part of the ultrasonic beam that passes through 
the weld nugget gives rise to principal echoes associated with the ultrasonic beam reflections which occur at the bottom surface of the lower sheet; whilst (ii) the part of the ultrasonic beam that does not pass through the weld nugget, and whose reflections occur at the interface between the two sheets, causes one-layer echoes between principal echoes.

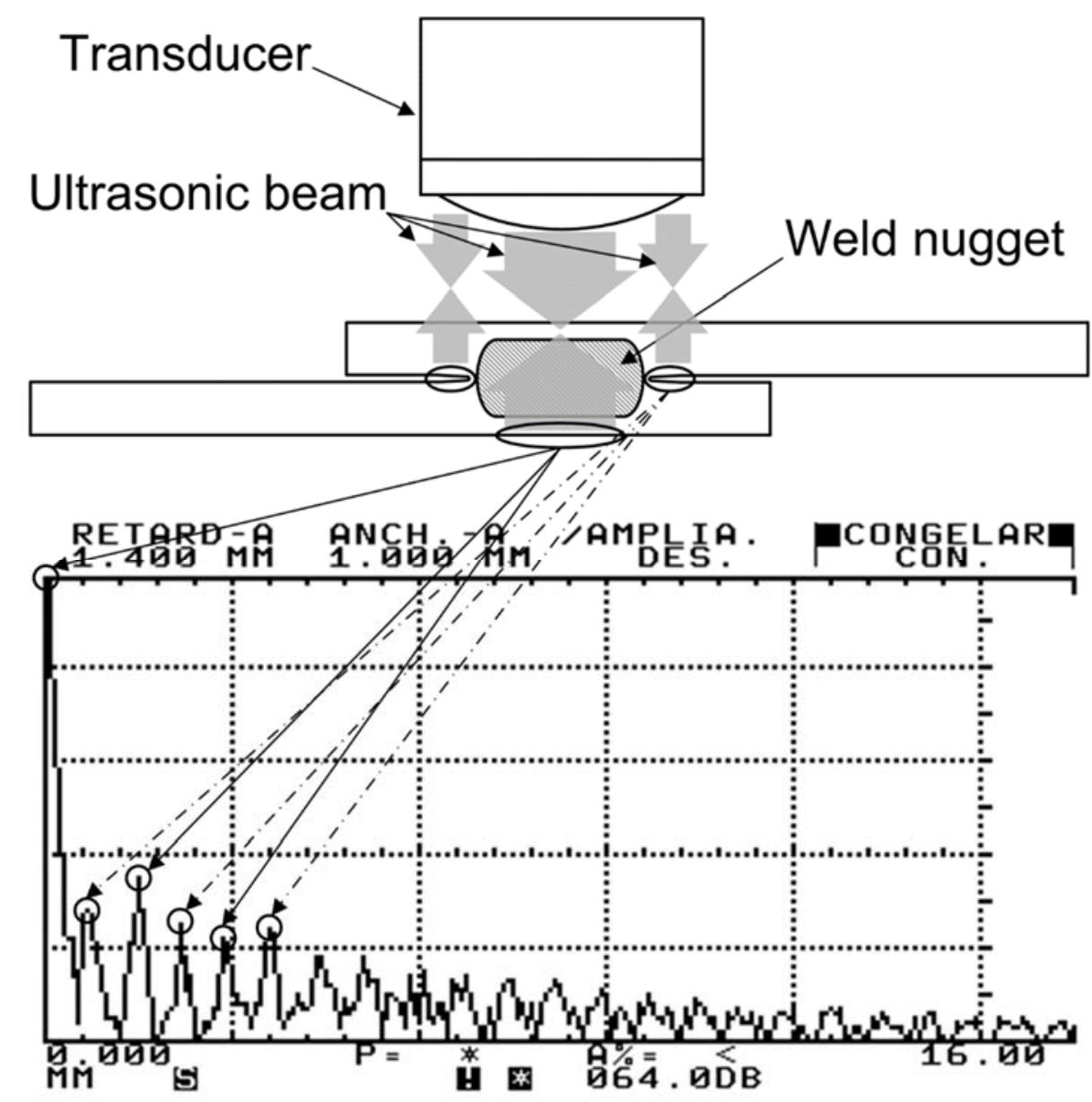

Fig. 3. Top: reflections of the ultrasonic beam for an unacceptable RSW joint in a tensile shear test specimen (not to scale); bottom: ultrasonic oscillogram of an unacceptable RSW joint.

Fig. 4 shows load vs. displacement curves obtained from the tensile-shear test performed on: (i) two tensile shear test specimens with acceptable RSW joints (one of which with a TSLBC value close to the threshold value); and (ii) two tensile shear test specimens with unacceptable RSW joints (one of which with a TSLBC value close to the threshold value). 


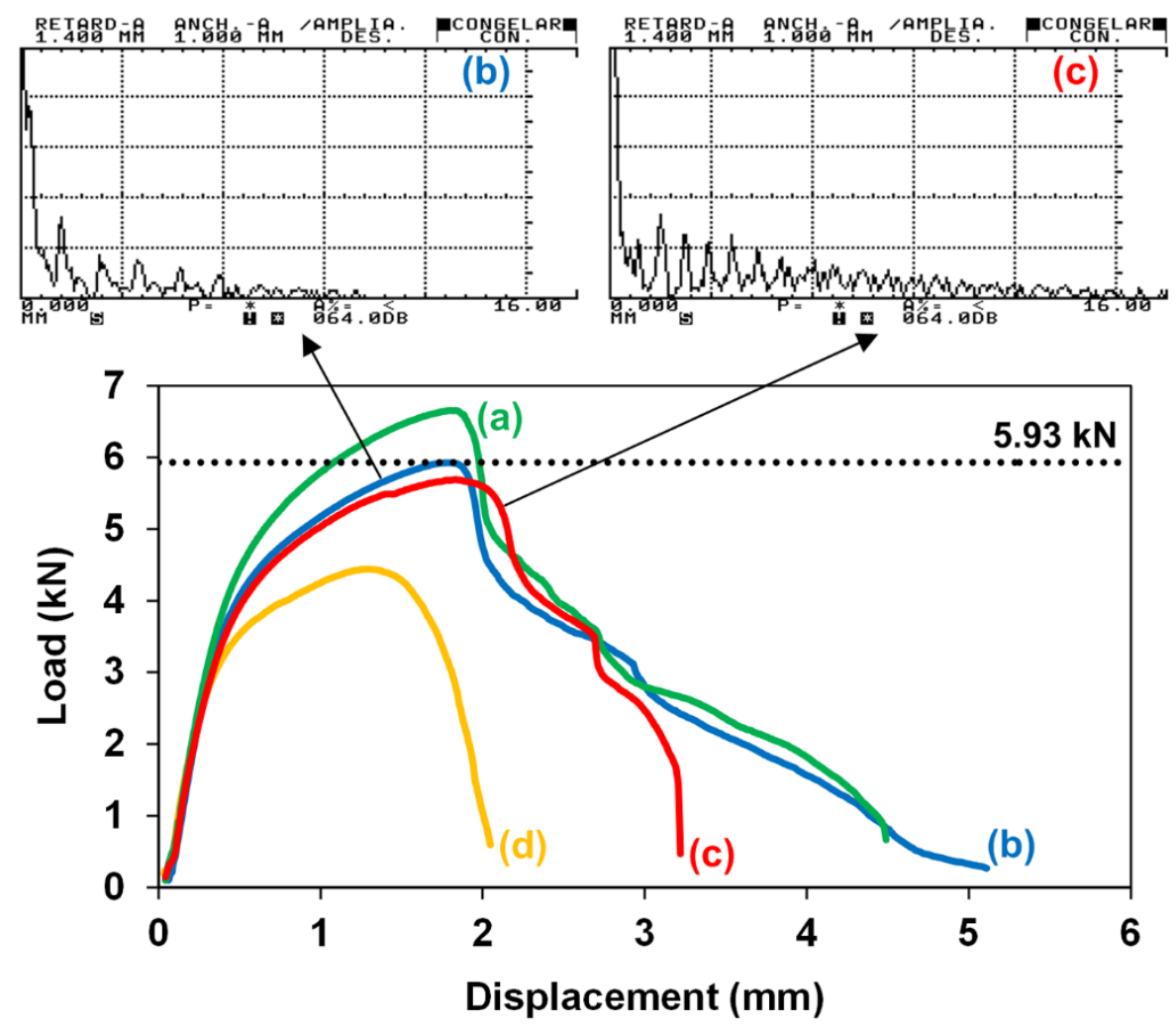

Fig. 4. Load vs. displacement curves obtained from the tensile-shear test performed on: (a) tensile shear test specimen with an acceptable RSW joint with TSLBC $=6.66 \mathrm{kN}$ (the corresponding ultrasonic oscillogram is shown in Fig. 2); (b) tensile shear test specimen with an acceptable RSW joint with TSLBC $=5.93 \mathrm{kN}$ (the corresponding ultrasonic oscillogram is indicated by an arrow); (c) tensile shear test specimen with an unacceptable RSW joint with TSLBC $=5.69 \mathrm{kN}$ (the corresponding ultrasonic oscillogram is indicated by an arrow); and (d) tensile shear test specimen with an unacceptable RSW joint with TSLBC $=4.44 \mathrm{kN}$ (the corresponding ultrasonic oscillogram is shown in Fig. 3).

\section{Theoretical background}

\subsection{Elastic Net regularization}

From a data analysis perspective, a regression problem is a supervised learning process for estimating the relationship between a continuous dependent output and a single or several predictor variables (discrete or continuous). This approach can be useful for several objectives such as identification of correlation relationships, the functional forms of these relationships or prediction of the output variable.

The most widely used regression technique is linear regression analysis, in which the output variable is assumed to be a linear combination of the predictors. Linear models are simple to describe and implement, having, therefore, advantages over other approaches in terms of interpretation and inference.

Linear regression has several extensions ranging from polynomial regression (one of the simplest) to smoothing splines and generalized additive models (GAMs), whose approach is more sophisticated as highlighted by James et al. [32]. All these extensions are based on the concept of basis functions, families of functions that are applied to the initial predictors in 
order to obtain extra predictors, so as to fit a linear model to the resulting predictors instead of the initial ones or to both.

Polynomial regression obtains the above-mentioned extra predictors by means of polynomial expansion of the initial predictive variables, performing a linear combination of both the initial and the polynomial predictors. This technique is potentially useful because it can capture nonlinear and interpretable relationships among variables that standard linear regression would miss. However, the obtained predictors are very correlated, and since the appropriate polynomial degree of the expansion for each variable is usually unknown, there is a danger of overfitting and capturing noise as signal, and consequently hinder predictive power or including spurious relationships that can make difficult the interpretation or control of the process.

An approach to overcome the problems of regression analysis is regularization. Regularization methods typically impose a penalty on the complexity of the fitted function. The most common regularization approaches are ridge (L2-regularization) proposed by Hoerl and Kennard [33] and lasso (L1-regularization) proposed by Tibshirani [34]. Ridge includes an additional penalization term to the ordinary least squares (OLS) estimate proportional to the sum of squares of the regression coefficients. Ridge is especially useful in the presence of high correlations between predictors, as in the case of polynomial regression, however, this penalization does not produce a parsimonious model since it does not remove predictors from the model. Lasso, on the contrary, imposes a penalty on the OLS estimate proportional to the sum of the absolute value of the regression coefficients. This method performs simultaneous shrinkage of the regression coefficients and automatic variable selection, resulting in simpler and more interpretable models. Fu [35] has shown that none of these regularization methods is universally better than the other, and that their results depend on the application context.

In this work, a more general regularization technique introduced by Zou and Hastie [25], the elastic net, is explored. Elastic net regularization combines L1 and L2 penalties introducing a second tuning parameter (see Eq. (2)). This double penalty includes ridge $(\alpha=1)$ and lasso $(\alpha=0)$ as particular cases. The quadratic part addresses high correlation potential problems among predictors while $\mathrm{L} 1$ generates a more parsimonious and sparse model through variable selection.

$$
\hat{\beta}=\operatorname{argmin}_{\beta}\left(\|y-\boldsymbol{X} \beta\|^{2}+\lambda\left[(1-\alpha)\|\beta\|_{1}+\alpha\|\beta\|^{2}\right]\right)
$$

\subsection{Smoothing splines}

Regression splines, the predecessors of smoothing splines, instead of fitting a high-degree polynomial over the entire range of predictors, involve dividing the range of predictors into distinct regions and fitting separate low-degree polynomials over these regions, including the necessary constraints so that the polynomials join smoothly at the region boundaries. Specifically, a degree- $d$ spline is a piecewise degree- $d$ polynomial with continuity in the derivatives up to degree $d-1$ at each split point (knot). This approach produces models with greater flexibility but that also tend to suffer from overfitting as pointed by James et al., [32]. Smoothing splines appear to overcome the problem of overfitting in regression splines by 
means of a "loss + penalty" formulation similar to the one from lasso and ridge, which aims at fitting data well but penalizes the excessive variability. Smoothing splines' formulation tries to find the function $g$ that minimizes:

$$
\sum_{i=1}^{n}\left(y_{i}-g\left(x_{i}\right)\right)^{2}+\lambda \int g^{\prime \prime}(t)^{2} d t
$$

Where $g^{\prime \prime}(t)$ is the second derivative of the model function, (which is a measure of its roughness: it is large in absolute value if $g(t)$ is very wiggly near $t$ and close to zero otherwise), and $\lambda$ is a nonnegative tuning parameter that controls the bias-variance trade-off.

It can be proved that the function $g(x)$ that minimizes Eq. (3) is a piecewise cubic polynomial with knots at every single observation and continuous first and second derivatives at each knot. Consequently, there is no need to choose the number of regions, being $\lambda$ the unique tuning parameter for the smoothing splines.

Smoothing splines are suitable for both univariate and multivariate problems. In univariate cases, the smoothing spline constitutes the whole model itself. As for multivariate case studies, in order to implement smoothing splines on these scenarios, it is necessary to resort to generalized additive models, (GAMs), which, as described by Hastie and Tibshirani [36], are an extension of multiple linear regression that allows smooth nonlinear functions (such as polynomials or smoothing splines) of each of the predictive variables while maintaining additivity of the effects of each predictor on the response. Since in this work, there is not a single predictor, the implementation of the smoothing splines has been done in the framework of a generalized additive model.

\subsection{Artificial Neural Networks}

The results of the elastic net and the GAM with smoothing splines have been compared to an ANN previously used by Martín et al. [19] which had very good performance.

A multilayer perceptron is a feedforward network of one or more layers of units -called neurons-. Each neuron works as a nonlinear function -called activation function- of the weighted sum of the set of input variables corresponding to the output values from the preceding layer plus a constant -called bias-, whose output is passed to the neurons of the following layer. Eq. (4) shows the output of the neuron $i$ in the layer $m ; w_{i j}^{(m-1)}$ is the weight that modulates the output $y_{j}^{(m-1)}$ of the neuron $j$ in the preceding layer $m-1 ; \theta_{i}^{(m)}$ is the bias; $N^{(m-1)}$ is the number of neurons in the layer $m$-1. The layer $m=1$ is not properly a layer of neurons because the output values are just the regressors of the model; the last layer corresponds to the dependent variable. The activation function $\phi(x)$ needs to be continuous, differentiable and monotonically increasing. Usually, all neurons use the same activation function (i.e. sigmoid functions), except for the input layer $(m=1)$ as it has already been explained, and the output layer whose output corresponds to a linear function $\phi(x)=x$. The rest of layers are called hidden layers. 


$$
y_{i}^{(m)}=\phi\left(\sum_{j=1}^{N^{(m-1)}} w_{i j}^{(m-1)} y_{j}^{(m-1)}+\theta_{i}^{(m)}\right)
$$

Traditionally, ANNs have used a backpropagation learning algorithm based on the gradientdescent method, but it is more common to apply other methods that increase the convergence speed, such as the Levenberg-Marquardt algorithm based on the hessian matrix.

ANNs are universal approximators [37] and provide good performance in many problems. Consequently, there are a lot of applications of ANNs in material science such as the work by Martin et al. [19] which was cited previously and that used ANNs to approximate the quality of RSW joints; or the work by Shirvanimoghaddam et al. [38] that uses ANNs to approximate the mechanical properties of composites, among other many examples.

However, it is important to highlight that ANNs have a significant disadvantage which is their lack of interpretability, because the information captured by the network during its training is not transparent to the user.

Martín et al. [19] applied a multilayer perceptron composed by multiple layers of neurons, where the transfer function for the hidden layers was the hyperbolic tangent function and the transfer function for the output layer was the identity function; the Levenberg-Marquardt algorithm, which can be considered a trust-region modification to Gauss-Newton, was used for training the ANN as explained by Hagan and Menhaj [39]. In the present work, the ANN was implemented with the $\mathrm{R}$ neuralnet package, that uses the resilient backpropagation without weight backtracking algorithm by Riedmiller and Braun [40] to estimate the parameters. Just as in the work of Martín et al., a set of networks with one and two hidden layers were tested (the inputs corresponding to the three predictors, i.e. WT, WC and EF, and the output was the TSLBC of the RSW joints). The error of the ANN has been computed by nested cross-validation (as well as the elastic net and smoothing spline models) to obtain comparable results.

\subsection{Nested cross-validation}

The regression models previously introduced require a prior phase to select some tuning parameters before fitting the data with the rest of the parameters. For example, the ANN needs to fix the number of hidden layers and the number of neurons in each layer before fitting data; the same applies to the penalization parameters of the elastic net model and the bias-variance parameter of the GAM with smoothing splines. Following the recommendations by Anderssen et al. [41] and Varma and Simon [42], nested cross-validation has been used for model selection and to estimate an unbiased error. In short, nested cross-validation consists on applying the cross-validation technique in two loops: an inner loop, which does not use all data, to select the tuning parameters and hence a model; and an outer loop, which includes the data that have not been used in the inner loop, to estimate the error of the selected model. 


\section{5 Comparison with other classifiers}

A further application of the regression models analyzed thus far in this paper is binary classification. By setting a threshold value, a regression model can also determine the class each observation belongs to.

In the present case study, the regression models with the lowest error rate were selected, and the threshold value was set at TSLBC $=5.93 \mathrm{kN}$, so that a RSW joint is acceptable if its TSLBC is greater or equal to $5.93 \mathrm{kN}$ and unacceptable otherwise.

In order to evaluate their performance as classifiers, a set of popular classifiers has been chosen as benchmark. In particular, the models analyzed in this paper have been compared with support vector machines using radial kernel, boosting and random forest techniques, because these classifiers offer the best performance in similar classification problems according to the conclusions of the work by Pereda et al. [6].

The assessment of the classification models has been done by means of nested crossvalidation. In order to ensure each fold is a good representative of the whole, stratified crossvalidation was implemented so as to have a balanced distribution of the classes in the different folds. As pointed by Kohavi [43], stratification is better in terms of both bias and variance when compared to regular cross-validation.

\section{Results and Discussion}

In the present work, so as to evaluate the proposed polynomial regression models with elastic net regularization, two particular implementations of them were made: one with quadratic polynomial expansion and another with cubic expansion. Firstly, their predictive accuracy was assessed and compared to the results obtained by two reference models: the ANN previously developed by Martin et al., [19], which was taken as baseline, and a GAM with smoothing splines over WC and WT, since according to James et al. [32], as GAMs allow nonlinear functions of the regressors, they exhibit greater flexibility and can, therefore, make more accurate predictions than standard linear models or its extensions based on expansions of the regressor space.

Table 3 summarizes the results for predictive accuracy given by the models mentioned above and table 4 is the summary of the one-way ANOVA test performed on them, which found the differences among the four models not statistically significant. As it can be seen in table 3 , the GAM with smoothing splines over WT and WC is the model with the lowest prediction error estimate, improving upon the results of the ANN. Regarding to the polynomial models, the results clearly state that the quadratic model outperforms the cubic one, which: (i) is coherent with the fact that a quadratic model is closer than a cubic one to the formula (Eq. (5)) that expresses the heat generated by Joule effect in the RSW process (this heat causes the formation and growth of the weld nugget [19], whose size is the most important factor affecting TSLBC $[9,10])$; and (ii) illustrates that more intricate models with greater number of parameters do not necessarily improve the results of simpler models. According to Aslanlar [26], the heat generated in the RSW process is: 


$$
Q=I^{2} R t
$$

where $Q$ is the heat generated $(J), I$ is the welding current (WC) (A), $R$ is the electrical resistance $(\Omega$ ) and $t$ is the welding time (WT) (s).

Table 3. Average prediction error (mean squared error), standard deviation (SD) and standard error (SE) for the elastic net regression models, the GAM with smoothing splines and the ANN.

\begin{tabular}{llll}
\hline Model description & Prediction Error Estimate & SD & SE \\
\hline Quadratic regression with elastic nets regularization & 0.10826 & 0.03685 & 0.01165 \\
Cubic regression with elastic nets regularization & 0.11062 & 0.03925 & 0.01241 \\
GAM with smoothing splines over WT and WC & 0.08627 & 0.02692 & 0.00851 \\
ANNs & 0.09445 & 0.03827 & 0.01210 \\
\hline
\end{tabular}

Table 4. Analysis of variance of Mean Square prediction Error.

\begin{tabular}{llllll}
\hline & Df & Sum sq & Mean sq & F value & $\operatorname{Pr}(>\mathrm{F})$ \\
\hline Model & 3 & 0.004 & 0.001334 & 1.049 & 0.383 \\
Residuals & 36 & 0.04579 & 0.001272 & & \\
\hline
\end{tabular}

From a RSW perspective further advantages of the quadratic regression model with elastic net regularization with respect to the GAM with smoothing splines are found in that (Fig. 5): (i) it captures the expulsion phenomenon caused, as pointed out by Aslanlar et al. [44], by an excessive heat input, which makes that for a given WC, if the WT is increased over a certain value, the obtained TSLBC starts decreasing instead of continuing increasing; (ii) the GAM with smoothing splines does not capture the expulsion phenomenon so well, however the prediction performance error in the areas of low WC is better; (iii) the GAM with smoothing splines evidences overfitting.

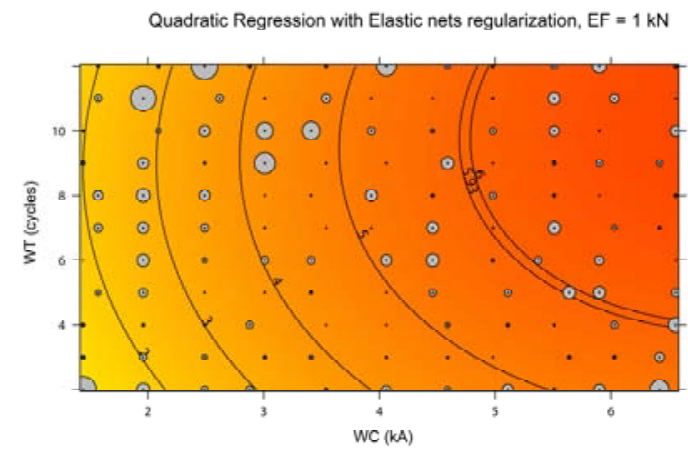

GAM with smoothing splines over WT and WC, EF $=1 \mathrm{kN}$

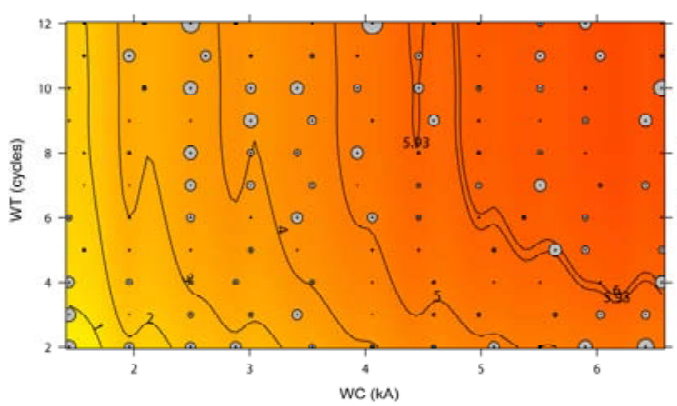

Quadratic Regression with Elastic nets regularization, $E F=1.5 \mathrm{kN}$

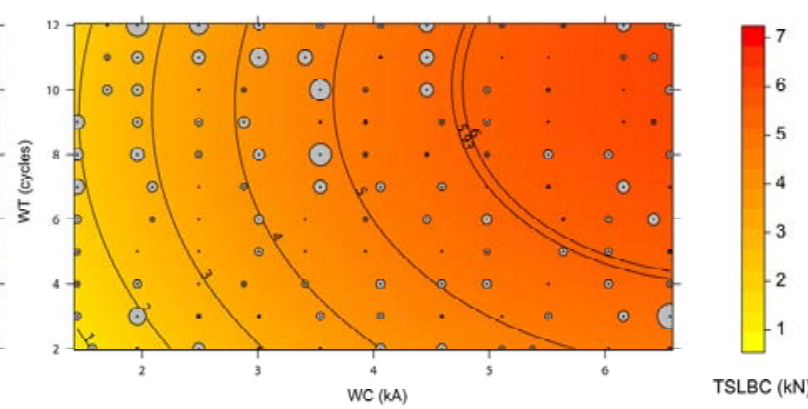

GAM with smoothing splines over $W T$ and $W C, E F=1.5 \mathrm{kN}$

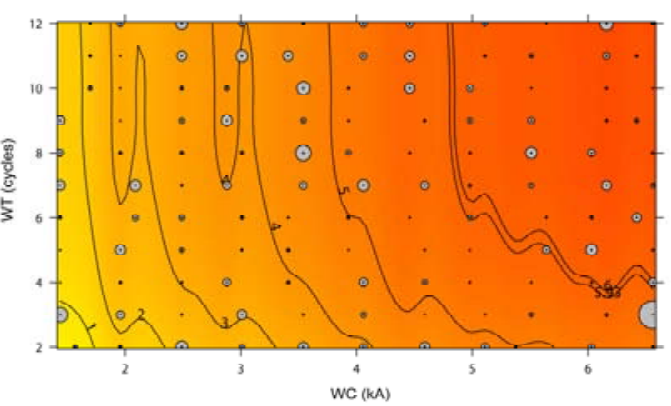

$1.0 \mathrm{kN}$

$0.6 \mathrm{kN}$

- $0.4 \mathrm{kN}$

- $0.2 \mathrm{kN}$

Error $(\mathrm{kN})$ 
Fig. 5. Matrix of TSLBC contour plots for the quadratic regression with elastic net regularization (top) and GAM with smoothing splines models (down), when $E F=1 \mathrm{kN}$ (left) and $\mathrm{EF}=1.5 \mathrm{kN}$ (right). Each plot displays the isolines of the TSLBC and the absolute prediction errors (grey circles) for each of the 242 RSW joints (the size of the circles is proportional to the error measured in $\mathrm{kN}$ ).

Given that in terms of statistical significance no-one of these four models is better than the rest and appealing to the principle of parsimony, it is justified to choose the quadratic regression model with elastic net regularization as the most adequate solution, since it provides an interesting trade-off between simplicity and interpretability of the model and good accuracy rate, exhibiting further advantages in terms of capturing better the RSW phenomena.

After selecting the model, it was trained on all the data of the RSW joints database, aiming at obtaining the best possible model by exploiting all the available data. Tables 5,6 and 7 give the details of the model obtained. Table 5 depicts the model coefficients, which give the model equation (6):

Table 5. Coefficients of the quadratic regression model with elastic net regularization trained on all the data.

\begin{tabular}{ll}
\hline Model regressors & Coefficient \\
\hline Intercept & -1.93914 \\
WT & 0.34904 \\
WT $^{2}$ & -0.02266 \\
WC & 1.95763 \\
WC & -0.14341 \\
EF & -0.36273 \\
WT·WC & 0.01260 \\
WT·EF & 0.03211 \\
WC.EF & 0.01422 \\
\hline
\end{tabular}

$$
\begin{gathered}
T S L B C=-1.93914+0.34904 \cdot W T-0.02266 \cdot W T^{2}+1.95763 \cdot W C-0.14341 \cdot W C^{2}-0.36273 \\
\cdot E F+0.01260 \cdot W T \cdot W C+0.03211 \cdot W T \cdot E F+0.01422 \cdot W C \cdot E F
\end{gathered}
$$

(6)

As for the model tuning parameters, they can be found in table 6 . In this particular case, the elastic net parameter alpha, in charge of modulating the trade-off between lasso and ridge penalization, takes a value of 0.01 , being really close to lasso penalization. However, a deeper analysis of the tuning parameter values obtained when performing nested cross-validation so as to select the model, evidences that generally lasso is not the case. Table 7 illustrates the average results over 10 folds for lambda and alpha. As it can be seen the average alpha value is 0.0755 , different from the 0 value of the lasso regularization. This is coherent with what Hastie et al. [25] highlighted, that normally the elastic net dominates the lasso by a good margin since the lasso is hurt by high correlation. Regarding the value of lambda, it is certainly low, which explains why although the model trained on all the data is close to a lasso penalization, no regressors have been eliminated. 
Table 6. Value of the model tuning parameters obtained via cross validation.

\begin{tabular}{ll}
\hline Tuning parameters selected via CV & Value \\
\hline Alpha & 0.01 \\
Lambda & 0.0006515474 \\
\hline
\end{tabular}

Table 7. Average value of the model tuning parameters obtained via nested cross-validation.

\begin{tabular}{lll}
\hline Values via NCV over ten folds & Average & SD \\
\hline Alpha & 0.0755 & 0.05965 \\
Lambda & 0.0007646 & 0.000295 \\
\hline
\end{tabular}

In a second step, the performance as classifier of the selected quadratic regression model with elastic net regularization was assessed. The comparison framework was constituted by the three benchmark classifiers suggested by Pereda et al. [6]: support vector machine with radial kernel, random forest and boosting, and by the GAM with smoothing splines over WT and WC used as classifier, which was taken under consideration since as it had the lowest predictive error in the previous analysis, a good performance as classifier could be expected too. Table 8 illustrates the results for classification. The lowest misclassification error was reached by boosting, followed closely by the GAM. The quadratic regression model has a misclassification error comparable to that of the random forest, which is lower than the one obtained with the support vector machine with radial kernel. Table 9 depicts the one-way ANOVA performed on the misclassification error estimates of the 5 classifiers. The results were found again not statistically significant, which implies that based on statistical significance it is not possible to establish one classifier as better than the others.

Table 8. Average Misclassification Error comparison.

\begin{tabular}{llll}
\hline Model description & Misclassification Error Estimate & SD & SE \\
\hline Quadratic regression with elastic nets regularization & 0.07370 & 0.04510 & 0.01426 \\
GAM with smoothing splines over WT and WC & 0.06933 & 0.05469 & 0.01730 \\
Random Forest & 0.07211 & 0.04476 & 0.01416 \\
Boosting & 0.06153 & 0.04388 & 0.01388 \\
Support Vector Machine with Radial Kernel & 0.09038 & 0.03624 & 0.01146 \\
\hline
\end{tabular}

Table 9. Analysis of variance of Misclassification Error

\begin{tabular}{llllll}
\hline & Df & Sum sq & Mean sq & F value & $\operatorname{Pr}(>\mathrm{F})$ \\
\hline Classifier & 4 & 0.00447 & 0.001118 & 0.545 & 0.704 \\
Residuals & 45 & 0.09241 & 0.002054 & & \\
\hline
\end{tabular}

Due to all the above, it can be asserted that the proposed quadratic regression model with elastic net regularization is good in terms of both predictive and classification accuracy, being comparable in terms of statistical significance to the best baseline models for both 
classification and predictive accuracy. This fact, together with the simplicity and interpretability of the model and its capability of capturing RSW phenomena such as material expulsion render it particularly suitable and interesting for RSW process design and quality control.

\section{Conclusions}

This work proposed polynomial expansion and elastic net regularization as regression technique for TSLBC prediction and quality control classification using the relevant welding parameters as inputs. The major conclusions are:

(1) Quadratic regression expansion with elastic net regularization exhibits good predictive accuracy providing at the same time simple and interpretable models, which is its main advantage over other techniques such as GAMs or ANNs that also have good predictive accuracy but lack explanatory power since they are "black boxes", i.e. the information captured by the model is no transparent to the user. This feature makes quadratic regression with elastic nets useful as decision support tool for establishing RSW operation conditions and for manufacturing control purposes, reducing post-welding testing

(2) The one-way analysis of variance conducted to test the average predictive performance of elastic net regularization, ANNs, and smoothing splines did not found any significant differences among them. In the computational experiments conducted in this paper the GAM with smoothing splines obtained the best results, improving the top prediction techniques in the literature, but the differences are not statistically significant.

(3) Binary classification accuracy results of quadratic regression with elastic net regularization are also found not significantly different from the state of the art RSW classifiers. However, the elastic nets, used as classification tool give an additional advantage over most traditional classifiers: it is possible to estimate the closeness of the assessed quality measure to the classification decision boundary, and consequently take a decision based on the criticality of the industrial process.

(4) Out of the range of application of the experimental data used in the present case the differences among the different mathematical models implemented may vary. Nevertheless, for the range of this data base which is adequate for the purpose of the present study, the conclusions obtained are robust.

\section{Acknowledgements}

The authors would like to thank Dr. L.R. Izquierdo for some advice and comments on this paper. The authors acknowledge support from the Spanish MICINN Project CSD2010-00034 (SimulPast CONSOLIDER-INGENIO 2010). 


\section{References}

[1] N. Becker, J. Gilgert, E.J. Petit, Z. Azari, The effect of galvanizing on the mechanical resistance and fatigue toughness of a spot welded assembly made of AISI410 martensite, Mater. Sci. Eng. A. 596 (2014) 145-156. doi:10.1016/j.msea.2013.12.008.

[2] F. Khodabakhshi, M. Kazeminezhad, A.H. Kokabi, Metallurgical characteristics and failure mode transition for dissimilar resistance spot welds between ultra-fine grained and coarse-grained low carbon steel sheets, Mater. Sci. Eng. A. 637 (2015) 12-22. doi:10.1016/j.msea.2015.04.019.

[3] D. Kianersi, A. Mostafaei, J. Mohammadi, Effect of welding current and time on the microstructure, mechanical characterizations, and fracture studies of resistance spot welding joints of AISI 316L austenitic stainless steel, Metall. Mater. Trans. A Phys. Metall. Mater. Sci. 45 (2014) 4423-4442. doi:10.1007/s11661-014-2421-z.

[4] Y. Feng, Y. Li, Z. Luo, Z. Ling, Z. Wang, Resistance spot welding of Mg to electro-galvanized steel with hot-dip galvanized steel interlayer, J. Mater. Process. Technol. 236 (2016) 114-122. doi:10.1016/j.jmatprotec.2016.05.015.

[5] O. Martín, M. López, F. Martín, Redes neuronales artificiales para la predicción de la calidad en soldadura por resistencia por puntos, Rev. Metal. 42 (2006) 345-353. doi:10.3989/revmetalm.2006.v42.i5.32.

[6] M. Pereda, J.I. Santos, Ó. Martín, J.M. Galán, Direct quality prediction in resistance spot welding process: Sensitivity, specificity and predictive accuracy comparative analysis, Sci. Technol. Weld. Join. 20 (2015) 679-685. doi:10.1179/1362171815Y.0000000052.

[7] D. Özyürek, An effect of weld current and weld atmosphere on the resistance spot weldability of 304L austenitic stainless steel, Mater. Des. 29 (2008) 597-603. doi:10.1016/j.matdes.2007.03.008.

[8] M. Zhou, S. Hu, H. Zhang, Critical specimen sizes for tensile-shear testing of steel sheets, Weld. J. 78 (1999) 305S-313S.

http://aws.org/wj/supplement/sept99/ZHOU.pdf.

[9] A. Hasanbasoglu, R. Kacar, Resistance spot weldability of dissimilar materials (AISI 316L-DIN EN 10130-99 steels), Mater. Des. 28 (2007) 1794- 
1800. doi:10.1016/j.matdes.2006.05.013.

[10] J.P. Kong, T.K. Han, K.G. Chin, B.G. Park, C.Y. Kang, Effect of boron content and welding current on the mechanical properties of electrical resistance spot welds in complex-phase steels, Mater. Des. 54 (2014) 598-609.

doi:10.1016/j.matdes.2013.08.098.

[11] R. Raoelison, A. Fuentes, P. Rogeon, P. Carré, T. Loulou, D. Carron, F. Dechalotte, Contact conditions on nugget development during resistance spot welding of $\mathrm{Zn}$ coated steel sheets using rounded tip electrodes, J. Mater. Process. Technol. 212 (2012) 1663-1669. doi:10.1016/j.jmatprotec.2012.03.009.

[12] D.H. Wolpert, The Lack of A Priori Distinctions Between Learning Algorithms, Neural Comput. 8 (1996) 1341-1390. doi:10.1162/neco.1996.8.7.1341.

[13] Ó. Martín, M. López, F. Martín, Artificial neural networks for quality control by ultrasonic testing in resistance spot welding, J. Mater. Process. Technol. 183 (2007) 226-233. doi:10.1016/j.jmatprotec.2006.10.011.

[14] W. Li, Manufacturing process diagnosis using functional regression, J. Mater. Process. Technol. 186 (2007) 323-330. doi:10.1016/j.jmatprotec.2006.12.052.

[15] H. Moshayedi, I. Sattari-Far, Numerical and experimental study of nugget size growth in resistance spot welding of austenitic stainless steels, J. Mater. Process. Technol. 212 (2012) 347-354. doi:10.1016/j.jmatprotec.2011.09.004.

[16] N. Ma, H. Murakawa, Numerical and experimental study on nugget formation in resistance spot welding for three pieces of high strength steel sheets, J. Mater. Process. Technol. 210 (2010) 2045-2052. doi:10.1016/j.jmatprotec.2010.07.025.

[17] L. Han, M. Thornton, D. Boomer, M. Shergold, A correlation study of mechanical strength of resistance spot welding of AA5754 aluminium alloy, J. Mater. Process. Technol. 211 (2011) 513-521. doi:10.1016/j.jmatprotec.2010.11.004.

[18] Y. Luo, W. Rui, X. Xie, Y. Zhu, Study on the nugget growth in single-phase AC resistance spot welding based on the calculation of dynamic resistance, J. Mater. Process. Technol. 229 (2016) 492-500. 
doi:10.1016/j.jmatprotec.2015.10.006.

[19] Ó. Martín, P. De Tiedra, M. López, M. San-Juan, C. García, F. Martín, Y. Blanco, Quality prediction of resistance spot welding joints of 304 austenitic stainless steel, Mater. Des. 30 (2009) 68-77.

doi:10.1016/j.matdes.2008.04.050.

[20] Ó. Martín, M. Pereda, J.I. Santos, J.M. Galán, Assessment of resistance spot welding quality based on ultrasonic testing and tree-based techniques, J. Mater. Process. Technol. 214 (2014) 2478-2487.

doi:10.1016/j.jmatprotec.2014.05.021.

[21] A. Vellido, J.D. Martin-Guerroro, P. Lisboa, Making machine learning models interpretable, in: 20th Eur. Symp. Artif. Neural Networks, Comput. Intell. Mach. Learn., 2012: pp. 163-172.

[22] C. Giraud-Carrier, Beyond predictive accuracy: what?, in: Proc. ECML-98 Work. Upgrad. Learn. to Meta-Level Model Sel. Data Transform., 1998: pp. 78-85.

[23] I. Bose, R.K. Mahapatra, Business data mining - a machine learning perspective, Inf. Manag. 39 (2001) 211-225. doi:10.1016/S03787206(01)00091-X.

[24] Y. Cho, S. Rhee, Primary Circuit Dynamic Resistance Monitoring and its Application to Quality Estimation during Resistance Spot Welding, Weld. J. 81 (2002) 104S-111S.

[25] H. Zou, T. Hastie, Regularization and variable selection via the elastic net, J. R. Stat. Soc. Ser. B (Statistical Methodol. 67 (2005) 301-320. doi:10.1111/j.1467-9868.2005.00503.x.

[26] S. Aslanlar, The effect of nucleus size on mechanical properties in electrical resistance spot welding of sheets used in automotive industry, Mater. Des. 27 (2006) 125-131. doi:10.1016/j.matdes.2004.09.025.

[27] ISO 14273, Specimen dimensions and procedure for shear testing resistance spot, seam and embossed projection welds, 2000.

[28] ASTM A 262-91, Standard practices for detecting susceptibility to intergranular attack in austenitic stainless steels, 1993. 
[29] P. Marashi, M. Pouranvari, S. Amirabdollahian, A. Abedi, M. Goodarzi, Microstructure and failure behavior of dissimilar resistance spot welds between low carbon galvanized and austenitic stainless steels, Mater. Sci. Eng. A. 480 (2008) 175-180. doi:10.1016/j.msea.2007.07.007.

[30] JIS Z 3140, Method of inspection for spot weld, 1989.

[31] T. Mansour, Ultrasonic testing of spot welds in thin gage steel, in: P. McIntire (Ed.), Nondestruct. Test. Handbook. Vol. 7 Ultrason. Test., American Society for Nondestructive Testing, Metals Park, OH, 1991: pp. 557-568.

[32] G. James, D. Witten, T. Hastie, R. Tibshirani, An Introduction to Statistical Learning, First, Springer Science \& Business Media, 2013. doi:10.1016/j.peva.2007.06.006.

[33] A.E. Hoerl, R.W. Kennard, Ridge Regression: Biased Estimation for Nonorthogonal Problems, Technometrics. 12 (1970) 55-67. doi:10.1080/00401706.1970.10488634.

[34] R. Tibshirani, Regression Selection and Shrinkage via the Lasso, J. R. Stat. Soc. Ser. B (Statistical Methodol. 58 (1996) 267-288. doi:10.2307/2346178.

[35] W.J. Fu, Penalized Regressions: The Bridge versus the Lasso, J. Comput. Graph. Stat. 7 (1998) 397. doi:10.2307/1390712.

[36] T.J. Hastie, R.J. Tibshirani, Generalized Additive Models, Chapman \& Hall/CRC Monographs on statistics and Applied Probability, New York, NY, 1990.

[37] K. Hornik, M. Stinchcombe, H. White, Multilayer feedforward networks are universal approximators, Neural Networks. 2 (1989) 359-366. doi:10.1016/0893-6080(89)90020-8.

[38] K. Shirvanimoghaddam, H. Khayyam, H. Abdizadeh, M. Karbalaei Akbari, A.H. Pakseresht, F. Abdi, A. Abbasi, M. Naebe, Effect of B4C, TiB2 and ZrSiO4 ceramic particles on mechanical properties of aluminium matrix composites: Experimental investigation and predictive modelling, Ceram. Int. 42 (2016) 6206-6220. doi:10.1016/j.ceramint.2015.12.181.

[39] M.T. Hagan, M.B. Menhaj, Training Feedforward Networks with the Marquardt Algorithm, IEEE Trans. Neural Networks. 5 (1994) 989-993. doi:10.1109/72.329697. 
[40] M. Riedmiller, H. Braun, A direct adaptive method for faster backpropagation learning: the RPROP algorithm, in: IEEE Int. Conf. Neural Networks, IEEE, 1994: pp. 586-591. doi:10.1109/ICNN.1993.298623.

[41] E. Anderssen, K. Dyrstad, F. Westad, H. Martens, Reducing over-optimism in variable selection by cross-model validation, Chemom. Intell. Lab. Syst. 84 (2006) 69-74. doi:10.1016/j.chemolab.2006.04.021.

[42] S. Varma, R. Simon, Bias in error estimation when using cross-validation for model selection, BMC Bioinformatics. 7 (2006) 91. doi:10.1186/1471-21057-91.

[43] R. Kohavi, A Study of Cross-Validation and Bootstrap for Accuracy Estimation and Model Selection, in: Int. Jt. Conf. Artifical Intell., Morgan Kaufmann Publishers, 1995: pp. 1137-1145.

[44] S. Aslanlar, A. Ogur, U. Ozsarac, E. Ilhan, Z. Demir, Effect of welding current on mechanical properties of galvanized chromided steel sheets in electrical resistance spot welding, Mater. Des. 28 (2007) 2-7. doi:10.1016/j.matdes.2005.06.022.

Figure Captions

Figure 1. (A) Micrograph that shows the cast dendritic microstructure of the weld nugget (WN) with coarser grains than the polygonal austenitic grains of the adjacent metal; electrolytic etching with oxalic acid according to Practice A of ASTM A 262-91:1993. (B) Diagram (not to scale) that shows the location of the area where the micrograph (A) was taken from the tensile shear test specimen.

Figure 2. Top: reflections of the ultrasonic beam for an acceptable RSW joint in a tensile shear test specimen (not to scale); bottom: ultrasonic oscillogram of an acceptable RSW joint.

Figure 3. Top: reflections of the ultrasonic beam for an unacceptable RSW joint in a tensile shear test specimen (not to scale); bottom: ultrasonic oscillogram of an unacceptable RSW joint.

Figure 4. Load vs. displacement curves obtained from the tensile-shear test performed on: (a) tensile shear test specimen with an acceptable RSW joint with TSLBC $=6.66 \mathrm{kN}$ (the corresponding ultrasonic oscillogram is shown in Fig. 2); (b) tensile shear test specimen with an acceptable RSW joint with TSLBC $=5.93 \mathrm{kN}$ (the corresponding ultrasonic oscillogram is indicated by an arrow); (c) tensile shear test specimen with an unacceptable 
RSW joint with TSLBC $=5.69 \mathrm{kN}$ (the corresponding ultrasonic oscillogram is indicated by an arrow); and (d) tensile shear test specimen with an unacceptable RSW joint with TSLBC $=4.44 \mathrm{kN}$ (the corresponding ultrasonic oscillogram is shown in Fig. 3).

Figure 5. Matrix of TSLBC contour plots for the quadratic regression with elastic net regularization (top) and GAM with smoothing splines models (down), when $E F=1 \mathrm{kN}$ (left) and EF=1.5 kN (right). Each plot displays the isolines of the TSLBC and the absolute prediction errors (grey circles) for each of the 242 RSW joints (the size of the circles is proportional to the error measured in kN).

Table Captions

Table 1. Chemical composition of the AISI 304 austenitic SS sheets (wt. \%).

Table 2. Mechanical properties of the AISI 304 austenitic SS sheets.

Table 3. Average prediction error (mean squared error), standard deviation (SD) and standard error (SE) for the elastic net regression models, the GAM with smoothing splines and the ANN.

Table 4. Analysis of variance of Mean Square prediction Error.

Table 5. Coefficients of the quadratic regression model with elastic net regularization trained on all the data.

Table 6. Value of the model tuning parameters obtained via cross validation.

Table 7. Average value of the model tuning parameters obtained via nested cross validation.

Table 8. Average Misclassification Error comparison.

Table 9. Analysis of variance of Misclassification Error 